\title{
MS12-01 | Exploring Cancer Heterogeneity: From DNA Mutability to Protein
}

\section{DYSFUNCTION}

Panchenko, Anna (Queen's University, Kingston, CAN)

Many different mutagenic processes cumulatively shape the observed somatic mutational trends in cancer and the growing body of evidence supports the dependence of mutation rates on local DNA sequence context and other factors. In our pursuit to decipher the mechanisms of cancer driver events, we develop methods to explore DNA context-dependent mutational patterns, identify underlying mutagenic processes related to infidelity of DNA replication and repair machinery, and various exogenic mutagenic factors. We derive mutagen and cancer-specific mutational background models and apply them to calculate expected DNA and protein site mutability to decouple relative contributions of mutagenesis and selection in carcinogenesis. Next we investigate molecular mechanisms of the impact of candidate driver missense mutations on protein structure and interactions and verify our predictions by different experimental approaches. 